\title{
IGCS20_1004
}

\section{IMMUNE CELL PROFILING MAY PREDICT CLINICAL OUTCOMES IN UTERINE LEIOMYOSARCOMA}

${ }^{1} \mathrm{~T}$ Fujii ${ }^{*},{ }^{1} \mathrm{H}$ Asano, ${ }^{2} \mathrm{R}$ Hasebe, ${ }^{3} \mathrm{~K}$ Hatanaka, ${ }^{4} \mathrm{~T}$ Saito, ${ }^{5} \mathrm{R}$ Matsuoka, ${ }^{1} \mathrm{M}$ Sakurai, ${ }^{1} \mathrm{H}$ Yamazaki, ${ }^{1} \mathrm{~K}$ Ihira, ${ }^{1} \mathrm{D}$ Emdo, ${ }^{1} \mathrm{~T}$ Mitamura, ${ }^{1} \mathrm{Y}$ Konno, ${ }^{1} \mathrm{~T}$ Kato, ${ }^{6} \mathrm{Y}$ Matsuno, ${ }^{2} \mathrm{M}$ Murakami, ${ }^{7} \mathrm{Y}$ Hatanaka, ${ }^{1} \mathrm{H}$ Watari. ${ }^{1}$ Department of Obstetrics and Gynecology, Faculty of Medicine and Graduate School of Medicine, Hokkaido University, Japan; ${ }^{2}$ Institute for Genetic Medicine, Hokkaido University, Japan; ${ }^{3}$ Clinical Research and Medical Innovation Center, Hokkaido University Hospital, Japan; ${ }^{4}$ RIKEN GENESIS CO., LTD., Japan; ${ }^{5}$ Department of Pathology, School of Medicine, International University of Health and Welfare, Japan; ${ }^{6}$ Department of Surgical Pathology, Hokkaido University Hospital, Japan; ${ }^{7}$ Research Division of Genome Companion Diagnostics, Hokkaido University Hospital, Japan

\subsection{6/ijgc-2020-IGCS.45}

Objectives Uterine leiomyosarcoma (uLMS) is one of rare and aggressive gynecologic malignancies. In this study, we aimed to correlate immune cell profiling with clinical outcomes in uLMS. Methods Total of 6 pathologically diagnosed uLMS patients were enrolled in this study. RNAs were extracted from FFPEs of normal and tumor lesions, and Cell Type Profiling analysis was conducted using nCounter, PanCancer Immune Profiling Panel (NanoString). For evaluating the related pathways, the RNAs were used to conduct microarray analysis using SurePrint G3 Human GE microarray $8 \times 60 \mathrm{~K}$ ver.2.0 (Agilent).

Results FIGO stage were IB in 4 patients, IIA in one patient, and IVB in one. We divided these patients into three groups by prognosis; good, no recurrence more than 2 years; intermediate, recurrent within 2 years; poor, recurrent immediately after surgery. In good prognostic group, CD8/regulatory $\mathrm{T}$ (Treg) ratio and Mast cell score in tumor lesions were elevated, and TP53 network, and DNA damage response signals were upregulated. In intermediate group, total TIL score in normal uterine were elevated, but $\mathrm{CD} 8 /$ Treg ratio was decreased in tumor lesion. And PI3K/AKT/mTOR signal and STAT3, IL8 signaling were upregulated. Finally, in poor prognostic group, total TIL score were decreased in normal and tumor lesions, and Neutrophil score was elevated in tumor lesions. Upregulation of DNA damage response but downregulated TP53 network.

Conclusions Immune cell profiling may predict clinical outcomes in uLMS. Further analyses, such as, correlation with signaling pathways affected to immune cell profiling in larger sample size are needed.
IGCS20_1008

\section{AN AUDIT OF THE COMPLETENESS OF THE PATHOLOGICAL REQUEST FORM FOR THE DIAGNOSIS OF POSSIBLE OVARIAN MALIGNANCY IN UK TERTIARY CANCER CENTRE}

${ }^{1} \mathrm{M}$ Daas*, ${ }^{2} \mathrm{~S}$ Addley, ${ }^{2} \mathrm{~S}$ Dhar, ${ }^{2} \mathrm{H}$ Soleymani Majd, ${ }^{2} \mathrm{M}$ Alazzam. ${ }^{1} J o r d a n$ University, Jordan; ${ }^{2}$ Oxford University Hospitals, UK

\subsection{6/ijgc-2020-IGCS.46}

Introduction Ovarian cancer prognosis and treatment plan are affected by the accuracy of the pathological report which highly depends on the clinical data. In this Audit, we reviewed the BGCS and The Royal College of Pathology guidelines for reporting ovarian carcinomas recommendation regarding the clinical information required on the pathological request and compare it with the current practice. This audit aims to enhance the pathological request-form of possible ovarian malignancy and increase the adherence of surgeons to the current guidelines.

Methods A retrospective study of consecutive 115 pathological request forms for patients who had undergone debulking surgeries for suspected ovarian malignancy at a tertiary university UK hospital. Pathology records were obtained from the 1st January 2016 until 31st December 2018.

Results $100 \%$ of the request forms contained the demographics, site of tumour origin and marked multiple specimens. However, there was no documentation for the clinical presentation, family history of cancers and history of previous hormonal therapy.

Implications Using generic pathology request forms resulted in missing key information which could very helpful to the pathologist in order to provide an accurate diagnosis. Electronic organ-specific pathology forms with dynamic fields must be incorporated into clinical practice for accurate diagnosis and staging. Key information such as clinical presentation, results of previous biopsies, radiological staging if available, and surgery details. Furthermore, add checkboxes for family history of cancers, history of taking any chemotherapy or hormonal replacement therapy.

\section{Abstract 47 Figure 1}

\section{THE CURRENT PRACTICE IN FILLING THE CLINICAL DATA}

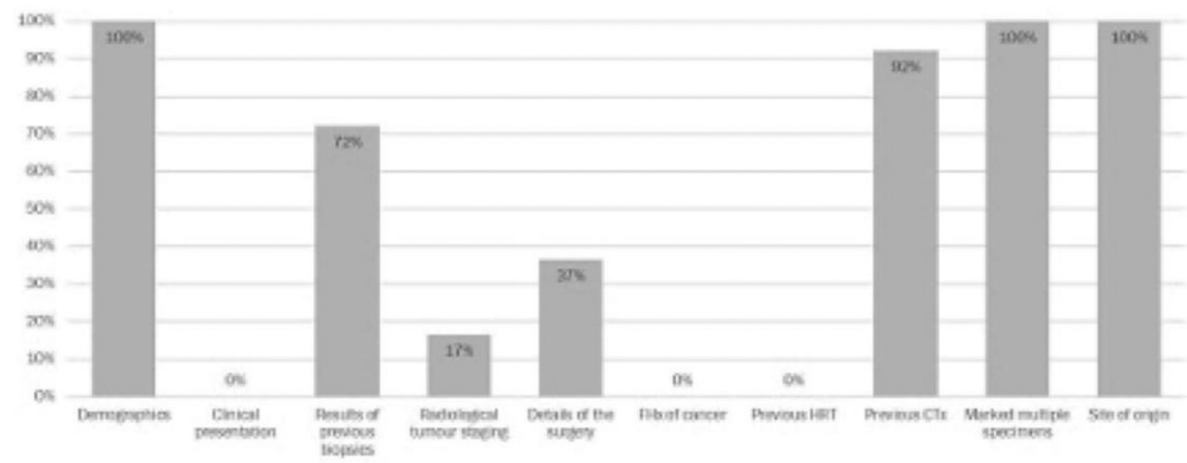

